\title{
Influence of Relative Humidity on Bubble-electrospun Nanofibers
}

\section{Shaokai $\mathrm{Li}^{1, \mathrm{a}}$, Fujuan $\mathrm{Liu}^{1,2^{\star}, \mathrm{b}}$, Yue Fang ${ }^{1, \mathrm{c}}$, Fangfang Zheng ${ }^{1, \mathrm{~d}}$, Junhua $\mathrm{Li}^{1, \mathrm{e}}$, Zhiru Chen ${ }^{3, f}$}

${ }^{1}$ National Engineering Laboratory for Modern Silk, College of Textile and Clothing Engineering, Soochow University, Suzhou, 215123, China

${ }^{2}$ Nantong Textile Institute, Soochow University, Nantong, 226019, China

${ }^{3}$ China Academy of Information and Communications Technology, Beijing, 100191, China

aemail: 1519678287@qq.com, bemail: liufujuan@suda.edu.cn, ${ }^{c}$ email:

1649934689@qq.com, 'email: 2194478865@qq.com, email: 1726457205@qq.com, 'email: chenzhiru@caict.ac.cn

Keywords: Relative Humidity; Nanofibers; Bubble-electrospinning

\begin{abstract}
Poly (vinyl alcohol) (PVA) and poly (vinyl pyrrolidone) (PVP) nanofibers prepared by bubble-electrospinning are studied experimentally. It shows that the relative humidity predominantly affect the morphology of the formed nanofibers. The nanofibers with high quality are essential for their applications in different natural environment.
\end{abstract}

\section{Introduction}

The nanofibers have many superior properties, such as superfine scale [1], large specific surface and porosity [2], high thermal and electric conductivity [3][4]. Therefore, they are widely applied in protective clothing [5], biomaterials [6], electronics [7], tissue scaffolds [8][9], filter and sensor materials [10][11], etc. There are lots of methods which can produce nanofibers. Electrospinning whatever solution or melt is an efficient method for forming nanofibers, and has attracted much interest. In recent years, bubble-electrospinning is a new method to produce nanofibers. The principle is that polymer bubble overcomes the surface tension to produce nanofibers with diameters ranging from $20 \mathrm{~nm}$ to several micrometers [12].

To our knowledge, bubble-electrospinning are greatly influenced by environmental factors. Various environmental conditions will directly affect the quality of the nanofibers. Recently there have been reports about the effect of temperature on the morphology of bubble-electrospun nanofibers. [12]. However, there is little research on relative humidity in the process of bubble-electrospinning.

In this paper, continuous nanofibers were prepared by bubble-electrospinning of poly(vinyl alcohol) (PVA) and poly(vinyl pyrrolidone) (PVP) solutions at different relative humidity from $35 \%$ to $70 \%$, respectively. The influence of relative humidity on the morphology and microstructures of nanofibers was studied while keeping other parameters constant.

\section{Experimental Part}

\section{Materials.}

PVA (98 \% hydrolyzed, average $\mathrm{Mw}=77,000 \mathrm{~g} / \mathrm{mol}$ ) was obtained from Sinopharm chemical Reagent Co., Ltd. (China) and PVP (K-30) provided by shanghai FeiHao trade incorporated company. PVA was dissolved into distilled water at $90{ }^{\circ} \mathrm{C}$ for $3 \mathrm{~h}$ with the concentration of $8 \%$ ( $\mathrm{g} / \mathrm{ml})$ under magnetic stirrer. PVP polymer solution with ethanol absolute as solvent was prepared at concentration of 35\%wt. All the chemicals were directly employed without further purification.

\section{Bubble-electrospinning.}

The bubble electrospinning device used was demonstrated schematically in the literature [13]. The applied voltage was kept at $20 \mathrm{kV}$, and the distance from the orifice to the collector for the bubble electrospun nanofibers depositing was set at $12 \mathrm{~cm}$. 


\section{SEM.}

The morphology of nanofibers was characterized by Scanning Electron Microscope (Hitachi S-4800, Tokyo, Japan) instrument. The nanofibrous membranes were sputter coated with a gold film before characterization. The fiber diameters were measured via Image J software (developed by the U.S. National Institutes of Health). At least 50 nanofibers in different SEM images were analyzed for each sample.

\section{Results and Discussion}

Figure 1 shows the process of bubble rupture in the bubble-electrospinning. In the beginning, the single bubble generated (Figure $1 \mathrm{a}$ ). As the role of high voltage electrostatic field, the bubble was drawn and stretched (Figure $1 \mathrm{~b}, \mathrm{c}$ and $\mathrm{d}$ ). When the electrostatic force overcomes the surface tension, the bubble bursts. A lot of jets were deposited in collecting plate and solidified to form nanofibers.

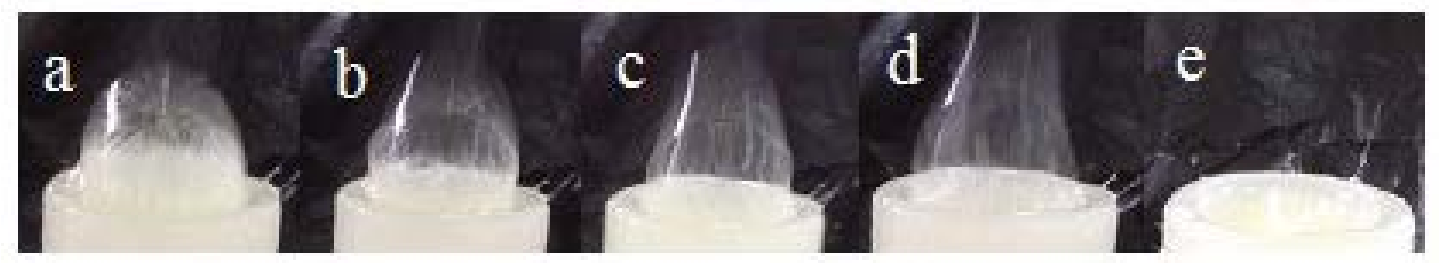

Fig. 1. The process of the bubble rupture
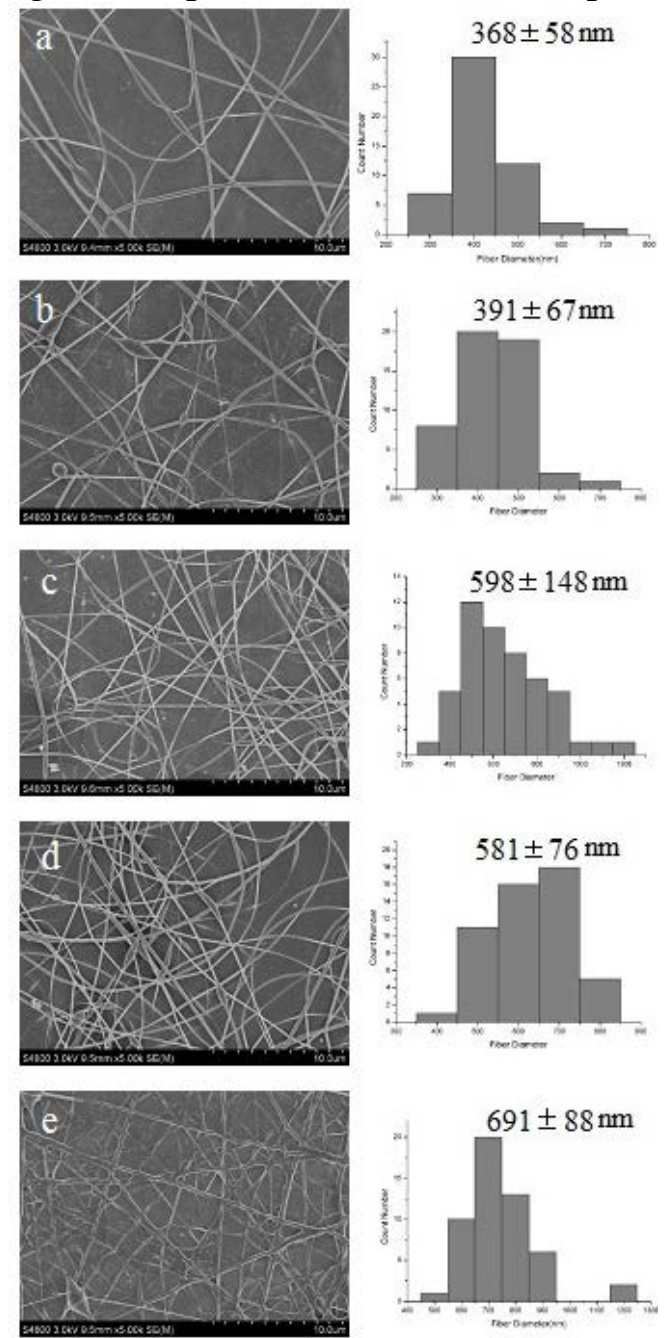

Fig.2. SEM micrographs and the diameter distribution histograms of the PVA nanofibers under the relative humidity of (a) 35\%, (b)40\%, (c)50\%, (d)60\%, (e)70\%. The diameter distribution histogram data are representative of independent experiments and all data are given as means \pm SD $(n=50)$ 
SEM micrographs of bubble-electrospun PVA nanofibers under different relative humidity (from $35 \%$ to $70 \%$ ) are exhibited in Figure 2. It is clear that the relative humidity below 60\% change did not appreciably affect the morphology of the PVA fibers. The smooth fibers were obtained in all cases (Figure $2 \mathrm{a}, \mathrm{b}$, c and d). When the relative humidity was up to $70 \%$, fiber adhesion appeared (Figure 2 e). Additionally, the fiber diameter was found to be 368, 391, 598, 581, and $691 \mathrm{~nm}$ at the relative humidity of $35,40,50,60$, and $70 \%$, respectively. The diameter of the fibers increases non-linearly with relative humidity. The diameter distribution histograms of the nanofibers formed under various relative humidity (Figure 2) show that the formed PVA fibers are relatively uniform.

Figure 3 exhibits SEM micrographs of bubble-electrospun PVP nanofibers under different relative humidity (from $35 \%$ to $70 \%$ ). Obviously, the relative humidity below $50 \%$ change did not appreciably affect the morphology and microstructures of the PVP fibers (Figure $3 \mathrm{a}$, b and c). When relative humidity reach $60 \%$, the fibers swelling and the diameter became larger (Figure $3 \mathrm{~d}$ ). Similarly as the relative humidity was up to $70 \%$, the fibers appeared severe adhesion (Figure 3 e). This is consistent with the previous experiment results of PVA.
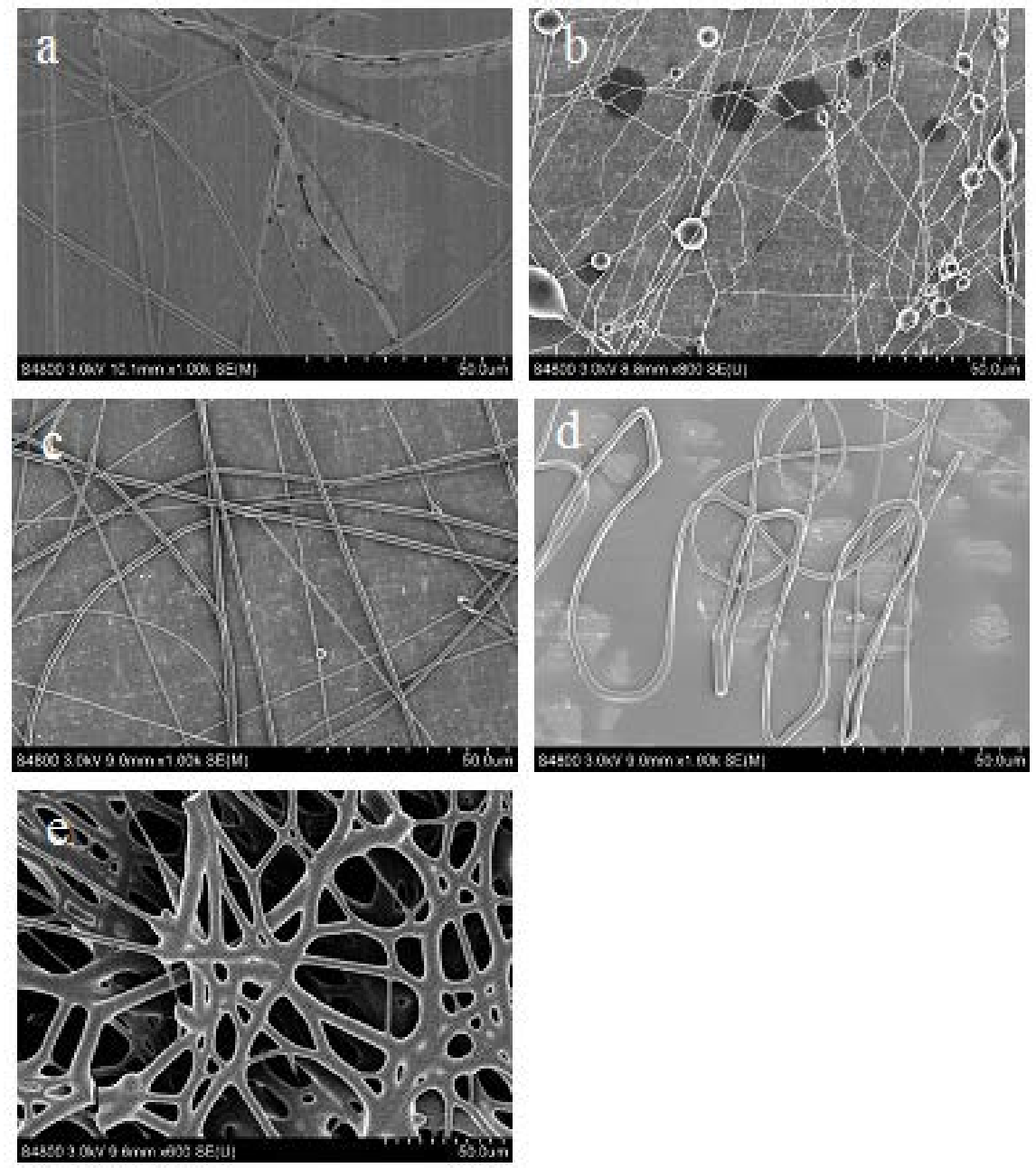

Fig.3. SEM micrographs of the PVP nanofibers under the relative humidity of (a) 35\%, (b)40\%, (c) $50 \%,(d) 60 \%,(e) 70 \%$

\section{Conclusions}

The influence of relative humidity on bubble-electrospun PVA and PVP nanofibers was investigated. At a certain condition, the morphology of nanofibers can be found relatively uniform. But when the relative humidity was up to $70 \%$, the fibers appeared adhesion which will restrict their applicability significantly. Therefore, the preparation of nanofibers with high quality under appropriate conditions are necessary. 


\section{Acknowledgement}

The work is supported by National Natural Science Foundation of China under grant No. 11372205, No. 51403143 and Science \& Technology Pillar Program of Jiangsu Province under grant No. BE2013072, Research and Innovation Project for College Graduates of Jiangsu Province under grant No. CXZZ13-0817, Natural Science Foundation of Jiangsu Province (Grant No. BK20140398), China Postdoctoral Science Foundation (Grant No. 2014M551658), Postdoctoral Research funding plan of Jiangsu Province (Grant No. 1302101B), Natural Science Foundation of the Jiangsu Higher Education Institutions of China (Grant No. 14KJA130001).

\section{References}

[1] Audrey Frenot, Ioannis S Chronakis. Polymer nanofibers assembled by electrospinning [J]. Current Opinion in Colloid \& Interface Science, 20038 (1) 64-75.

[2] Xinli Zhu, Wenguo Cui, Xiaohong Li, Yan Jin. Electrospun fibrous mats with high porosity as potential scaffolds for skin tissue engineering [J]. Biomacromolecules, 20089 (7) 1795-1801.

[3] Farzad Dabirian, Seyed Abdolkarim Hosseini Ravandi, Juan P Hinestroza, Ramazan Ali Abuzade. Conformal coating of yarns and wires with electrospun nanofibers [J]. Polymer Engineering and Science, 201252 (8) 1724-1732.

[4] M. Gorji, Ali. A. A. Jeddi, A. A. Gharehaghaji. Fabrication and characterization of Polyurethane electrospun nanofiber membranes for protective clothing application [J]. Journal of Applied Polymer Science, 2012125 (5) 4135-4141.

[5] El-Refaie Kenawy, Gary L. Bowlin, Kevin Mansfield, John Layman, David G. Simpson, Elliot H. Sanders, Gary E. Wnek. Release of tetracycline hydrochloride from electrospun poly(ethylene-co-vinylacetate), poly(lactic acid), and a blend [J]. Journal of Controlled Release, 200281 (1-2) 57-64.

[6] Ian D. Norris, Manal M. Shaker, Frank K. Ko, Alan G. MacDiarmid. Electrostatic fabrication of ultrafine conducting fibers: polyaniline/polyethylene oxide blends [J]. Synthetic Metals, 2000 114 (2) 109-114.

[7] H. Yoshimotoa, Y. M. Shina, H. Teraia, J. P. Vacanti. A biodegradable nanofiber scaffold by electrospinning and its potential for bone tissue engineering [J]. Biomaterials, 200324 (12) 2077-2082.

[8] Z. M. Huang, Y. Z. Zhang, M. Kotakli, S. Ramakrishna. A review on polymer nanofibers by electrospinning and their applications in nanocomposites [J]. Composites Science \& Technology, 200363 (15) 2223-2253.

[9] Soo-Hyoung Lee, Bon-Cheol Ku, X. Wang, L. A. Samuelson, J. Kumar. Design, synthesis and electrospinning of a novel fluorescent polymer for optical sensor applications [J]. Materials Research Society Symposium, 2002708 403-408.

[10]Peter P. Tsaia, Heidi Schreuder-Gibson, Phillip Gibson. Different electrostatic methods for making electret filters [J]. Journal of Electrostatics, 200254 (3-4) 333-341.

[11] Qiuna Cui, Ya Li, Fujuan Liu. Effect of temperature on the morphology of bubble-electrospun nanofibers [J]. Thermal Science, 201418 (5) 1707-1709.

[12] Shaokai Li, Fujuan Liu, Fangfang Zheng, Yue Fang, Junhua Li, Jihuan He. Effect of ultrasonic vibration on the morphology of bubble-electrospun nanofibers [J]. Fibers and Polymers, 201516 (11) $2432-2436$. 